\title{
Scatter Correction for Positron Emission Mammography
}

\author{
Jinyi Qi and Ronald H. Huesman \\ Center for Functional Imaging, Lawrence Berkeley National Laboratory, Berkeley, \\ CA 94720, USA. \\ E-mail: $\{$ jqi,rhhuesman\}@lbl.gov
}

\begin{abstract}
In this paper we present a scatter correction method for a regularized list mode maximum likelihood reconstruction algorithm for the positron emission mammograph (PEM) that is being developed at our laboratory. The scatter events inside the object are modeled as additive Poisson random variables in the forward model of the reconstruction algorithm. The mean scatter sinogram is estimated using a Monte Carlo simulation program. With the assumption that the background activity is nearly uniform, the Monte Carlo scatter simulation only needs to run once for each PEM configuration. This saves computational time. The crystal scatters are modeled as a shift-invariant blurring in image domain because they are more localized. Thus, the useful information in the crystal scatters can be deconvolved in high-resolution reconstructions. The propagation of the noise from the estimated scatter sinogram into the reconstruction is analyzed theoretically. The results provide an easy way to calculate the required number of events in the Monte Carlo scatter simulation for a given noise level in the image. The analysis is also applicable to other scatter estimation methods, provided that the covariance of the estimated scatter sinogram is available.
\end{abstract}

\section{Introduction}

A rectangular positron emission tomograph (PEM), dedicated to imaging the human breast, is under development at our Laboratory [1]. The tomograph consists of four banks of detector modules (two banks of $3 \times 3$ modules left and right and two banks of $3 \times 4$ modules top and bottom). Each module consists of an $8 \times 8$ array of $3 \times 3 \times 30$ $\mathrm{mm}^{3}$ lutetium oxyorthosilicate (LSO) crystals. The maximum field of view (FOV) of the system is $96 \times 72 \times 72 \mathrm{~mm}^{3}$. The LSO crystals are coupled to a photo-diode (PD) array at the front and a photo multiplier tube (PMT) at the back. By measuring the signal from both the PD and the PMT, the depth of interaction (DOI) of the photon can be estimated [2]. For each crystal, the system digitizes the DOI of the photon with three bits. Each detector is placed in coincidence with all detectors in the other three banks, giving rise to 172 million possible lines of response (LORs). The system operates exclusively in fully 3D mode.

The data from the new tomograph are stored in list mode format because the total number of detections is generally far less than the total number of LORs. A 
list mode likelihood reconstruction algorithm has been developed to reconstruction the data with explicit modeling of the DOI information in the forward projection matrix [3]. Here we present a scatter correction method for this list mode likelihood reconstruction algorithm. The scatter events inside the object are modeled as additive Poisson random variables in the likelihood function. The expectation of the scatters is estimated before the reconstruction starts. A Monte Carlo simulation method is used to estimate the scatter sinogram; however, the reconstructions method can use the scatter sinogram estimated from other approaches as well. The scatter events inside detector crystal are modeled as a shift invariant blurring in the image domain because they are relatively localized to the original source. Unlike the traditional convolution-subtraction methods [4], this blurring operator is incorporated in the forward model of the reconstruction algorithm. The noise propagation from the Monte Carlo scatter sinogram into the reconstructed image has also been theoretically analyzed. Such analysis is useful in determining the total number of events that is required for the Monte Carlo scatter simulation.

\section{Theory}

\subsection{List Mode Likelihood Reconstruction with Scatter Correction}

Histogrammed PET data are generally modeled as a collection of independent Poisson random variables. By treating the detections in each LOR separately, we can derive the appropriate log-likelihood function for list mode data [3]:

$$
L(\boldsymbol{x})=\sum_{k=1}^{K} \log \left[\sum_{j=1}^{N} p\left(i_{k}, j\right) x_{j}+s_{i_{k}}\right]-\sum_{j=1}^{N} \varepsilon_{j} x_{j},
$$

where $x_{j}$ is mean activity inside the $j$ th voxel of the unknown image, $p(i, j)$ is the probability of detecting an event from the $j$ th voxel in the $i$ th LOR, $s_{i}$ is the expectation of the scatter in the $i$ th LOR, $i_{k}$ is the index of the LOR of the $k$ th detection, $\varepsilon_{j} \equiv \sum_{i} p(i, j), K$ is the total number of detections, and $N$ is the total number of image voxels. $s_{i}$ has to be estimated before the reconstruction.

The maximum likelihood (ML) estimate can be found by maximizing (1). A popular ML algorithm for PET reconstruction is the expectation maximization (EM) algorithm $[5,6,7]$. However, the ML solution is noisy. Hence some form of regularization (or prior function) is needed to reconstruct a reasonable image. The prior function used in [3] is a Gaussian prior whose logarithm is of the form

$$
\beta U(\boldsymbol{x})=\frac{\beta}{2}(\boldsymbol{x}-\boldsymbol{m})^{\prime} \boldsymbol{R}(\boldsymbol{x}-\boldsymbol{m}),
$$

where $\beta$ is the smoothing parameter, $\boldsymbol{m}$ is the estimated mean of the unknown image, and $\boldsymbol{R}$ is a positive definite (or semidefinite) matrix.

Combining the likelihood function (1) and the image prior (2), the reconstruction is found as

$$
\hat{\boldsymbol{x}}=\arg \max _{\boldsymbol{x} \geq 0}[L(\boldsymbol{x})-\beta U(\boldsymbol{x})] .
$$


For further simplification, $\boldsymbol{R}$ is chosen to be a diagonal matrix, so the EM algorithm can be used to solve (3). The EM update equation is [3]

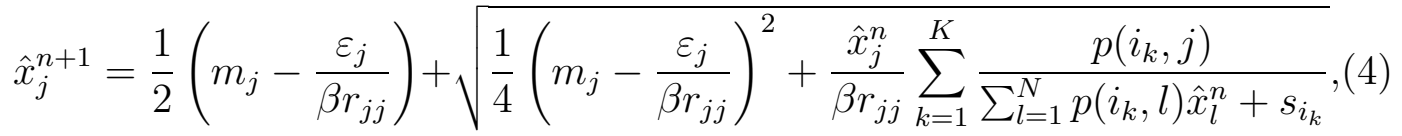

where $r_{j j}$ is the $(j, j)$ th element of $\boldsymbol{R}$.

\subsection{Estimate Mean Scatter Sinogram using Monte Carlo Simulation}

The reconstruction method described above requires the mean scatter sinogram be known beforehand. For conventional PET systems, a scatter sinogram can be estimated using the convolution method [4], the hardware dual energy windows method [8], the Monte Carlo simulation method [9], and analytical methods [10, 11]. Among these methods, the Monte Carlo simulation can model multiple scatters and various effects in the photon detection process. Thus, it can achieve high accuracy, albeit time consuming. In addition, most other scatter estimation methods require fitting a computed scatter sinogram to the tails of the emission sinogram that consist of pure scatter events. This is not practical for the PEM geometry, as the whole FOV is filled with activity. Therefore, we adopt the Monte Carlo method here.

The Monte Carlo scatter simulation requires both emission and attenuation maps. They are generally obtained from an initial reconstruction without scatter correction. One advantage of breast imaging with FDG is that the background is quite uniform. If we can assume that the whole FOV is filled with uniform activity and that features such as cancerous lesions account for a small fraction of the radioactivity, then we may only need to run the Monte Carlo simulation once for each system configuration, which can save a large amount of computational time. A potential problem is that in real situations the breast may not fill the whole FOV even by applying some compression using the top and bottom detector banks. In this case we will need first to estimate the support of the breast (either from projection data or an initial reconstruction) and then run the Monte Carlo simulation to estimate the scatter sinogram. When time is limited, we can either smooth the Monte Carlo scatter sinogram to reduce its variance, or use the image based scatter estimation algorithm in [11] instead.

We used the same Monte Carlo simulation program that we developed for generating list mode data to estimate the scatter sinogram. The program traces all $511 \mathrm{keV}$ photon pairs randomly generated inside the FOV. For each photon, it first computes the interaction point based on the attenuation length, and then it determines whether it is a photoelectric or Compton interaction. If it is photo-electric, it dumps all of its current energy; if it is Compton, it computes the deposited energy and the new direction of the photon using the Klein-Nishima formula, and it continues to trace the photon until the photon has dumped all its energy or traveled outside of the system. A photon is detected when the energy deposited at one detector is greater than a preselected threshold. A coincidence event is recorded if both photons are detected. The Monte Carlo simulation program histograms the scattered and unscattered (true) events separately. In the 
current implementation we have not used any variance reduction techniques or other acceleration methods.

For each individual data set, the scatter sinogram is then estimated by

$$
\hat{s}_{i}=\frac{\text { total events in the data }}{\text { total events in Monte Carlo simulation }} s_{i}^{M C} \text {, }
$$

where $s_{i}^{M C}$ is the number of scatter events in the $i$ th LOR in the Monte Carlo simulation. This assumes the scatter fraction and profile in each data set are the same, as most scatters are generated from the uniform background.

\subsection{Noise Propagation}

Noise is inevitable in the Monte Carlo scatter sinogram due to the Poisson nature of the counting process. The longer the simulation runs, the less noise there is. This presents a tradeoff between time and accuracy. In this section we analyze how the noise in the scatter sinogram propagates into the reconstruction.

We denote the MAP reconstruction in (3) as $\hat{\boldsymbol{x}}(\boldsymbol{y}, \hat{\boldsymbol{s}})$ to indicate that $\hat{\boldsymbol{x}}$ is dependent on estimated scatter sinogram $\hat{\boldsymbol{s}}$, where $\boldsymbol{y}$ denotes the collection of the detected events. We approximate $\hat{\boldsymbol{x}}(\boldsymbol{y}, \hat{\boldsymbol{s}})$ using a first order Taylor series expansion at the point $(\boldsymbol{y}, \hat{\boldsymbol{s}})=(\overline{\boldsymbol{y}}, \boldsymbol{s})$, where $\overline{\boldsymbol{y}}$ is the expectation of $\boldsymbol{y}$

$$
\hat{\boldsymbol{x}}(\boldsymbol{y}, \hat{\boldsymbol{s}}) \approx \hat{\boldsymbol{x}}(\overline{\boldsymbol{y}}, \boldsymbol{s})+\nabla_{s} \hat{\boldsymbol{x}}(\overline{\boldsymbol{y}}, \boldsymbol{s})(\hat{\boldsymbol{s}}-\boldsymbol{s})+\nabla_{y} \hat{\boldsymbol{x}}(\overline{\boldsymbol{y}}, \boldsymbol{s})(\boldsymbol{y}-\overline{\boldsymbol{y}}) .
$$

From (6), we have the following expression for the covariance of noise in the reconstruction caused by the noise in the estimated scatter sinogram

$$
\Sigma^{s}(\hat{\boldsymbol{x}}) \approx \nabla_{s} \hat{\boldsymbol{x}}(\overline{\boldsymbol{y}}, \boldsymbol{s}) \Sigma(\hat{\boldsymbol{s}})\left[\nabla_{s} \hat{\boldsymbol{x}}(\overline{\boldsymbol{y}}, \boldsymbol{s})\right]^{\prime},
$$

where $\Sigma(\hat{\boldsymbol{s}})$ is the covariance matrix of the estimated scatter sinogram.

To compute $\nabla_{s} \hat{\boldsymbol{x}}(\boldsymbol{y}, \boldsymbol{s})$, we follow the idea presented in [12]. We restrict our attention to the situations where the solution of (3) satisfies

$$
0=\left.\frac{\partial}{\partial x_{j}}[L(\boldsymbol{y} \mid \boldsymbol{x}, \boldsymbol{s})-\beta U(\boldsymbol{x})]\right|_{\boldsymbol{x}=\hat{\boldsymbol{x}}(\boldsymbol{y}, \boldsymbol{s})}, \quad j=1, \ldots, N .
$$

While this assumption precludes inequality constraints, it should work here because of the uniform background. Differentiating (8) with respect to $s_{i}$ by applying the chain rule and solving the resulting equation, we get

$$
\begin{aligned}
\nabla_{s} \hat{\boldsymbol{x}}(\boldsymbol{y}, \boldsymbol{s})= & \left\{-\left.\nabla_{x x}[L(\boldsymbol{y} \mid \boldsymbol{x}, \boldsymbol{s})-\beta U(\boldsymbol{x})]\right|_{\boldsymbol{x}=\hat{\boldsymbol{x}}(\boldsymbol{y}, \boldsymbol{s})}\right\}^{-1} \\
& \left.\nabla_{x s}[L(\boldsymbol{y} \mid \boldsymbol{x}, \boldsymbol{s})-\beta U(\boldsymbol{x})]\right|_{\boldsymbol{x}=\hat{\boldsymbol{x}}(\boldsymbol{y}, \boldsymbol{s})}
\end{aligned}
$$

where the $(j, k)$ th element of the operator $\nabla_{x x}$ is $\frac{\partial^{2}}{\partial x_{j} \partial x_{k}}$, and the $(j, l)$ th element of the operator $\nabla_{x s}$ is $\frac{\partial^{2}}{\partial x_{j} \partial s_{l}}$.

From (1) and (2), we can derive

$$
-\nabla_{x x}[L(\boldsymbol{y} \mid \boldsymbol{x}, \boldsymbol{s})-\beta U(\boldsymbol{x})]=\boldsymbol{P}^{\prime} \operatorname{diag}\left[\frac{y_{i}}{(\boldsymbol{P} \boldsymbol{x}+\boldsymbol{s})_{i}^{2}}\right] \boldsymbol{P}+\beta \boldsymbol{R}
$$


and

$$
\nabla_{x s}[L(\boldsymbol{y} \mid \boldsymbol{x}, \boldsymbol{s})-\beta U(\boldsymbol{x})]=\boldsymbol{P}^{\prime} \operatorname{diag}\left[\frac{-y_{i}}{(\boldsymbol{P} \boldsymbol{x}+\boldsymbol{s})_{i}^{2}}\right]
$$

Then

$$
\begin{aligned}
\nabla_{s} \hat{\boldsymbol{x}}(\overline{\boldsymbol{y}}, \boldsymbol{s})= & \left\{\boldsymbol{P}^{\prime} \operatorname{diag}\left[\frac{\bar{y}_{i}}{(\boldsymbol{P} \hat{\boldsymbol{x}}+\boldsymbol{s})_{i}^{2}}\right] \boldsymbol{P}+\beta \boldsymbol{R}\right\}^{-1} \\
& \boldsymbol{P}^{\prime} \operatorname{diag}\left[\frac{-\bar{y}_{i}}{(\boldsymbol{P} \hat{\boldsymbol{x}}+\boldsymbol{s})_{i}^{2}}\right]
\end{aligned}
$$

Substituting (10) into (7) results in

$$
\begin{aligned}
\Sigma^{s}(\hat{\boldsymbol{x}}) \approx & \left\{\boldsymbol{P}^{\prime} \operatorname{diag}\left[\frac{\bar{y}_{i}}{(\boldsymbol{P} \hat{\boldsymbol{x}}+\boldsymbol{s})_{i}^{2}}\right] \boldsymbol{P}+\beta \boldsymbol{R}\right\}^{-1} \\
& \boldsymbol{P}^{\prime} \operatorname{diag}\left[\frac{\bar{y}_{i}}{(\boldsymbol{P} \hat{\boldsymbol{x}}+\boldsymbol{s})_{i}^{2}}\right] \Sigma(\hat{\boldsymbol{s}}) \operatorname{diag}\left[\frac{\bar{y}_{i}}{(\boldsymbol{P} \hat{\boldsymbol{x}}+\boldsymbol{s})_{i}^{2}}\right] \boldsymbol{P} \\
& \left\{\boldsymbol{P}^{\prime} \operatorname{diag}\left[\frac{\bar{y}_{i}}{(\boldsymbol{P} \hat{\boldsymbol{x}}+\boldsymbol{s})_{i}^{2}}\right] \boldsymbol{P}+\beta \boldsymbol{R}\right\}^{-1} .
\end{aligned}
$$

In general, $\hat{\boldsymbol{x}}$ is a slightly blurred version of $\boldsymbol{x}$, so the projection $\boldsymbol{P} \hat{\boldsymbol{x}}+\boldsymbol{s}$ is approximately equal to the mean of the data, $\overline{\boldsymbol{y}}$. Therefore, we can simplify the above expression to

$$
\Sigma^{s}(\hat{\boldsymbol{x}}) \approx[\boldsymbol{F}+\beta \boldsymbol{R}]^{-1} \boldsymbol{P}^{\prime} \operatorname{diag}\left[\frac{\sigma_{\hat{s}_{i}}^{2}}{\bar{y}_{i}^{2}}\right] \boldsymbol{P}[\boldsymbol{F}+\beta \boldsymbol{R}]^{-1},
$$

where $\boldsymbol{F}=\boldsymbol{P}^{\prime} \operatorname{diag}\left[\frac{1}{\bar{y}_{i}}\right] \boldsymbol{P}$ is the Fisher information matrix and $\sigma_{\hat{s}_{i}}^{2}$ is the variance of $\hat{s}_{i}$. Eq. (12) is the covariance matrix of the noise in the reconstruction that is propagated from the estimated scatter sinogram.

The covariance of noise in the reconstruction caused by the Poisson noise in the data is $[12]$

$$
\begin{aligned}
\Sigma^{y}(\hat{\boldsymbol{x}}) & \approx \nabla_{y} \hat{\boldsymbol{x}}(\overline{\boldsymbol{y}}, \boldsymbol{s}) \Sigma(\boldsymbol{y})\left[\nabla_{y} \hat{\boldsymbol{x}}(\overline{\boldsymbol{y}}, \boldsymbol{s})\right]^{\prime} \\
& \approx[\boldsymbol{F}+\beta \boldsymbol{R}]^{-1} \boldsymbol{F}[\boldsymbol{F}+\beta \boldsymbol{R}]^{-1}
\end{aligned}
$$

Adding (12) and (13), we get the covariance of the total noise

$$
\Sigma_{\text {total }}(\hat{\boldsymbol{x}})=[\boldsymbol{F}+\beta \boldsymbol{R}]^{-1} \boldsymbol{P}^{\prime} \operatorname{diag}\left[\frac{\sigma_{\hat{s}_{i}}^{2}}{\bar{y}_{i}^{2}}+\frac{1}{\bar{y}_{i}}\right] \boldsymbol{P}[\boldsymbol{F}+\beta \boldsymbol{R}]^{-1} .
$$

It shows that the noise from the scatter sinogram is equivalent to an increase of the noise in the data by a factor of $1+\sigma_{\hat{s}_{i}}^{2} / \bar{y}_{i}$, where $\sigma_{\hat{s}_{i}}^{2} / \bar{y}_{i}$ is equal to the scatter fraction of the $i$ th LOR divided by the ratio between the total number of detections in the Monte Carlo simulation and the total number of detections in the data. For example, if the average scatter fraction is $30 \%$, and the Monte Carlo simulation has 30 times as many events as the data, then the increase of the variance in reconstruction caused by the scatter sinogram will be about $1 \%$. Thus, (14) can be used to determine the number of events required in the Monte Carlo simulation and to design better simulation strategy. 


\subsection{Modeling of Crystal Scatters}

Monte Carlo simulation shows that about 33\% of detected events have undergone Compton scatter inside LSO crystal but not inside the object (see Section 3). We call these events "crystal scatters." Although we can model crystal scatters the same way as scatters inside the object, we believe that crystal scatters carry more useful information than scatters inside the FOV because the attenuation length of the LSO crystal is much shorter than that of the soft tissue. Photons scattered inside a crystal are usually detected by nearby detectors. Thus, the mispositioning of crystal scatters is relatively local to the original source. This local property provides us with the opportunity to extract useful information from crystal scatters.

For histogrammed data, the crystal scatters can be modeled using a sinogram blurring function $[13,14]$. However, list mode reconstruction precludes us from using the sinogram approach. Therefore, we propose the following method. The detectors used in the PEM scanner are capable of measuring depth of interaction information and hence can achieve nearly isotropic resolution in all three dimensions. We then can assume that the detection response function of a $511 \mathrm{keV}$ photon is shift invariant for photons that have the same incident angle and rotation invariant for photons with different incident angles. With this assumption we can model the mean of crystal scatters using a shift-invariant spherically symmetric blurring function in image space; i.e., the mean detection for each LOR is now

$$
y_{i}=\sum_{j} p(i, j)[\boldsymbol{H} \boldsymbol{x}]_{j}+s_{i},
$$

where $\boldsymbol{H}$ is the blurring function used to model crystal scatters and $s_{i}$ is the mean contribution of scatters from the object. $\boldsymbol{H} \boldsymbol{x}$ can be easily computed using the Fast Fourier Transform, since $\boldsymbol{H}$ is a shift-invariant operator. The model in (15) is quite

similar to the one that Reader et al. [15] used to model finite resolution effects in list mode reconstruction.

Bailey and Meikle [4] proposed modeling scatters as a convolution of the true projection in the sinogram domain. Some other researchers have also modeled scatters as a convolution of the real image $[16,17]$. Our approach differs from these work in several respects. The convolution in the previous authors' methods was mainly used to model object scatters, and the scatters were subtracted from the reconstructed image; whereas here the blurring function is used to model crystal scatters, and scatters are corrected using deconvolution to restore high resolution. Because soft tissue has a long attenuation length, object scatters do not have the local property compared to crystal scatters. Hence, we think (15) is more suitable for modeling crystal scatters than object scatters. 


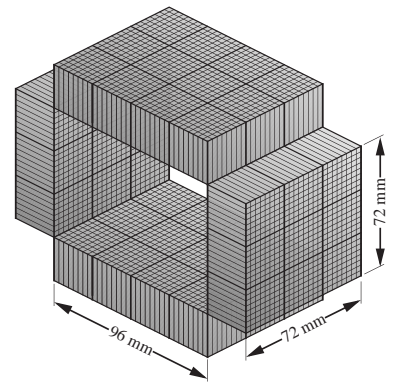

(a)

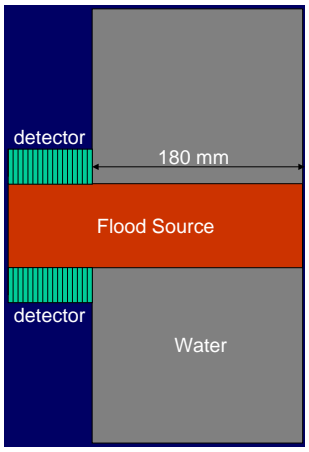

(b)

Figure 1. (a) The rectangular PEM geometry. (b) Simulation setup for studying scatter. Uniform flood source $\left(96 \times 72 \times 252 \mathrm{~mm}^{3}\right)$ extending out of FOV by $180 \mathrm{~mm}$ in axial direction is surrounded by scatter medium (water) $\left(372 \times 372 \times 180 \mathrm{~mm}^{3}\right)$.

\section{Simulation Results}

\subsection{Scatter Distribution}

The Monte Carlo simulation uses the appropriate energy-dependent cross sections for the interaction of photons in water (in the field of view) and in the LSO detector. Fig. 1a shows the rectangular geometry of the PEM scanner. In the simulations we did not model the energy resolution of the detectors (i.e., the energy resolution was perfect). It means that we could reject all scatter events if we had used an energy threshold of $511 \mathrm{keV}$. An energy threshold $(270 \mathrm{keV})$ that was lower than that expected for the real scanner (typically $350 \mathrm{keV}$ ) was used to partially compensate the perfect energy resolution. With only a flood source inside the FOV, the average detection efficiency was about $13 \%$ for the energy threshold of $270 \mathrm{keV}$ (there was no upper limit). Of all the detected events, there were about 35\% unscattered events, $32 \%$ events scattered in the FOV, and 33\% events scattered in the detector (not scattered in the FOV).

For breast imaging, there is a significant portion of activity outside of FOV. The out-of-FOV activity contributes to both randoms and scatters. Here we study the scatter events from the activity out-of-FOV using the setup shown in Fig. 1b. The uniform flood source is about $25 \mathrm{~cm}$ long in the axial direction. Fig. 2 shows the scatter fraction image obtained from the Monte Carlo simulation. The voxel intensities represent the fraction of the events originating from each voxel that are scattered and detected. With the out-of-FOV activity and scatter medium, more scatter events are detected. The extra scatters originate from both inside and outside the FOV, as shown in Fig. 2c. The overall increase of the scatter events is about $7.7 \%$ (relative to the number of the scatter events without out-of-FOV object), which is relatively small. Thus, we choose to ignore the out-of-FOV activity in the scatter correction. 


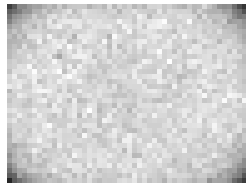

(a)

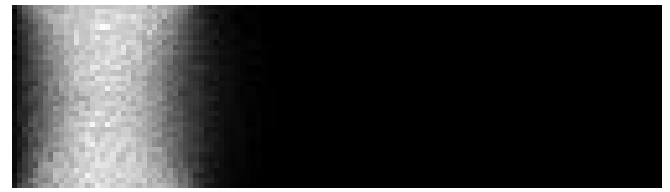

(b)

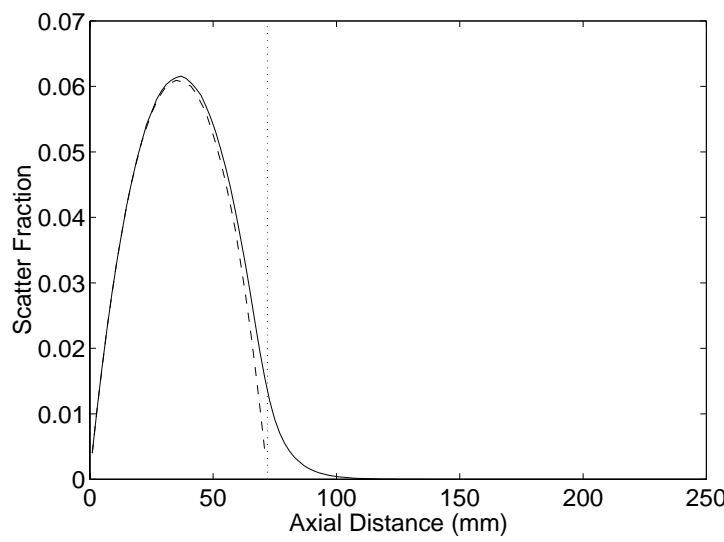

(c)

Figure 2. The front view center slice (a) and the side view center slice (b) of the scatter fraction image. The voxel intensities represent the fraction of the events originating from each voxel that are scattered and detected (not the image reconstructed from scatter events). (c) Plot of the average scatter fraction as a function of the axial distance from the front of the scanner. The dashed line denotes the fraction of scatter without out-of-FOV activity and scatter medium. The dotted line indicates the axial limit of the PEM scanner.

\subsection{Scatter Correction}

We simulate a subject weighing $70 \mathrm{~kg}$ and an injection of $1 \mathrm{mCi}$ of FDG, which is uniformly distributed within the body. This activity density within the $72 \times 72 \times 96 \mathrm{~mm}^{3}$ field of view and an imaging time of $60 \mathrm{~s}$ gives about 16 million disintegrations within the imaging volume, resulting in about 2 million detected events.

Fig. 3 shows an example of a reconstruction of a simulated flood source with and without scatter correction. The reconstructed image without scatter correction (Fig. 3a) shows brighter at the center of the FOV and darker at the corners in the front view slice. The scatter-corrected image (Fig. 3b) shows more uniform activity distribution and reflects the correct activity level.

\subsection{Crystal Scatters}

3.3.1. Computing the Blurring Kernel We chose eight points to study crystal scatters. For each point, we generated Monte Carlo simulated data of a point source with only crystal scatters (no scatter in FOV) and reconstructed using the list mode ML algorithm without any scatter modeling. The reconstructed point source images are shown in Fig. 4. The three views are the front view, side view and top view of the plane through 


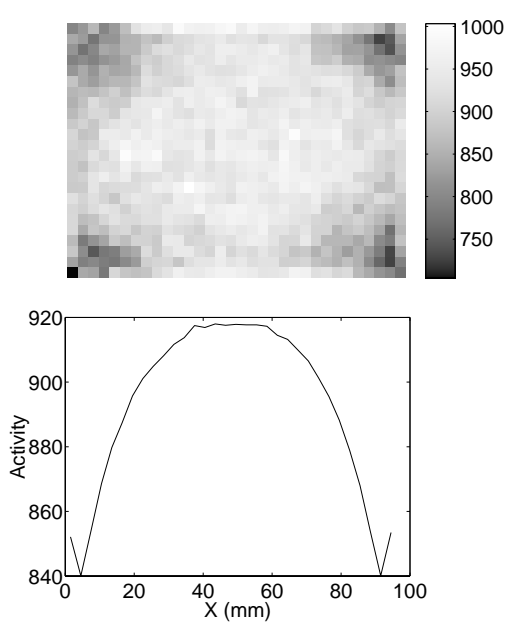

(a)
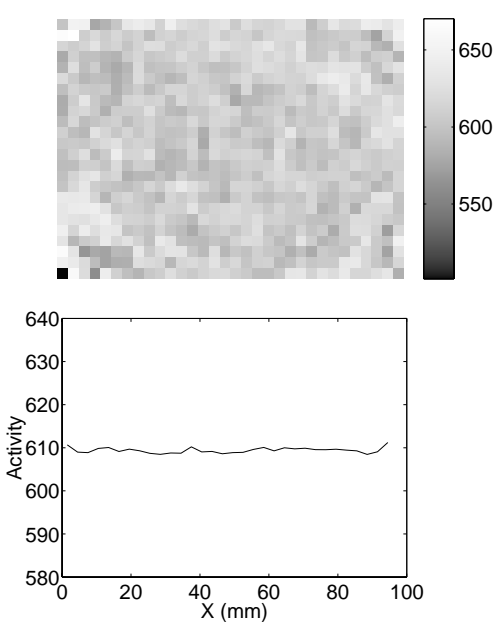

(b)

Figure 3. Front view slices and the average horizontal profiles of the simulated reconstructions: (a) without scatter correction; (b) with scatter correction. The scatter-corrected image shows a more uniform activity distribution and reflects the correct activity level. Note that the gray level maps in (a) and (b) are different.

the point source. The point spread functions are close to being spherically symmetric and shift invariant. We fitted the point spread functions with a sum of two exponentials

$$
h(r)=a_{1} \exp \left(-\lambda_{1} r\right)+a_{2} \exp \left(-\lambda_{2} r\right),
$$

where $r$ is the distance from the point source.

Fig. 5 shows the fitting function with the comparison to the measured results from each reconstructed point source image. The measured profiles were obtained by averaging the intensities of all the voxels within a certain range of distance from the point source. It shows that the proposed function $\left(a_{1}=.9943, \lambda_{1}=3.8891, a_{2}=.0063\right.$, $\left.\lambda_{2}=.5567\right)$ has a good fit for all point spread functions at least up to the $20 \mathrm{~mm}$ radius, which also confirms the previous observation that these point spread functions are close to shift invariant.

\subsubsection{Reconstructions Using the blurring function $h(r)$ we obtained, we reconstructed} a point source on a warm background. In Fig. 6 we plotted the contrast recovery coefficient (CRC) of the point source vs. background noise curves with and without modeling of the crystal scatters. CRC is defined as the ratio between the measured contrast and the true contrast. The points were obtained using different prior parameters. Here we used the gamma prior [18] because the EM update equation (4) for Gaussian prior is very sensitive to round-off errors when $\beta r_{j j}$ is small. The results show that by modeling the crystal scatters we can achieve a higher contrast at the same background noise level compared to the reconstruction without the modeling. It also shows that the improvement is more pronounced when the contrast is high. 
$(49,37,37)$
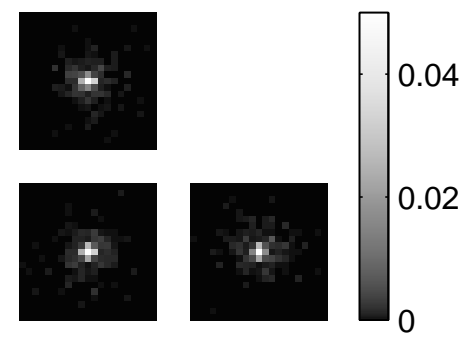

$(49,37,19)$
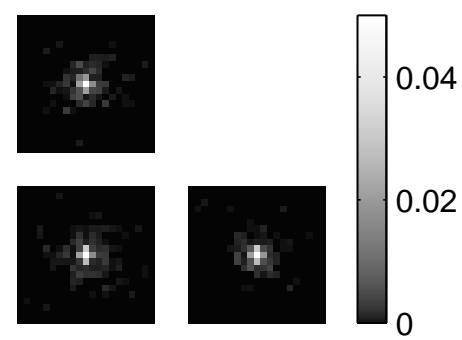

$(25,19,37)$
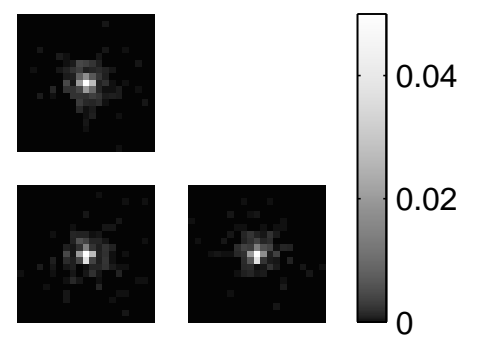

$(25,37,37)$
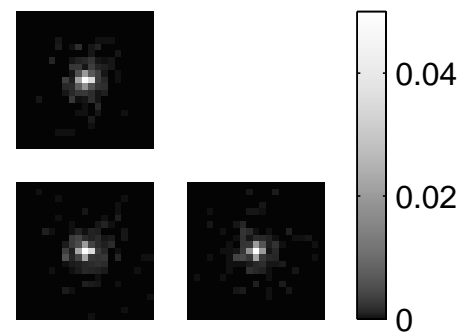

$(25,37,19)$
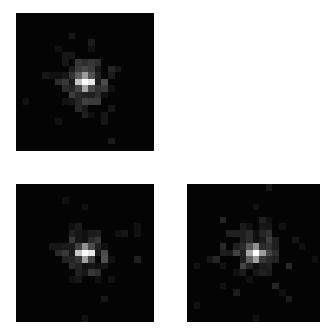

$(25,19,19)$
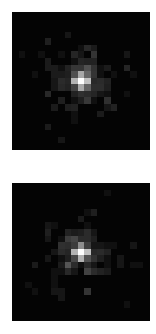

$(49,19,37)$

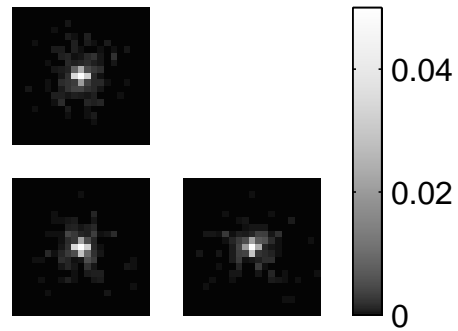

$(49,19,19)$

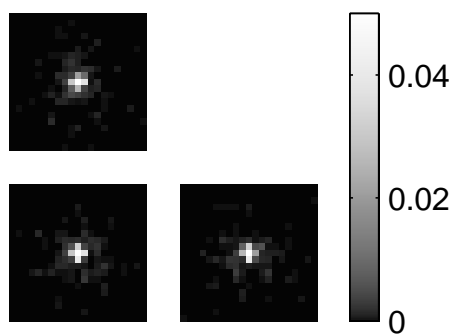

Figure 4. The reconstructed point source images with crystal scatters. The numbers above each image are the $(\mathrm{x}, \mathrm{y}, \mathrm{z})$ coordinates of the point source. The three views are the top view (top), front view (lower left), and side view (lower right) through the point source. In each image the values are normalized with respect to the voxel that contains the point source and are thresholded at 0.05 .

\section{Conclusion}

We have implemented a scatter correction method for the list mode likelihood reconstruction algorithm for the PEM and have presented simulation results. The object scatters are modeled as additive Poisson random variables in the forward projection. The specific application of PEM allows us to assume that the background emission activity and attenuation are quite uniform. This simplifies the the Monte Carlo scatter simulation. The crystal scatters are modeled using a shift invariant blurring function in image space, assuming that the detector can achieve isotropic resolution by measuring DOI information. Reconstructions of computer simulated data show that by modeling of crystal scatters we can achieve higher contrast at any given background noise level compare with the reconstruction without the modeling. The improvement is more pronounced at high resolution. 

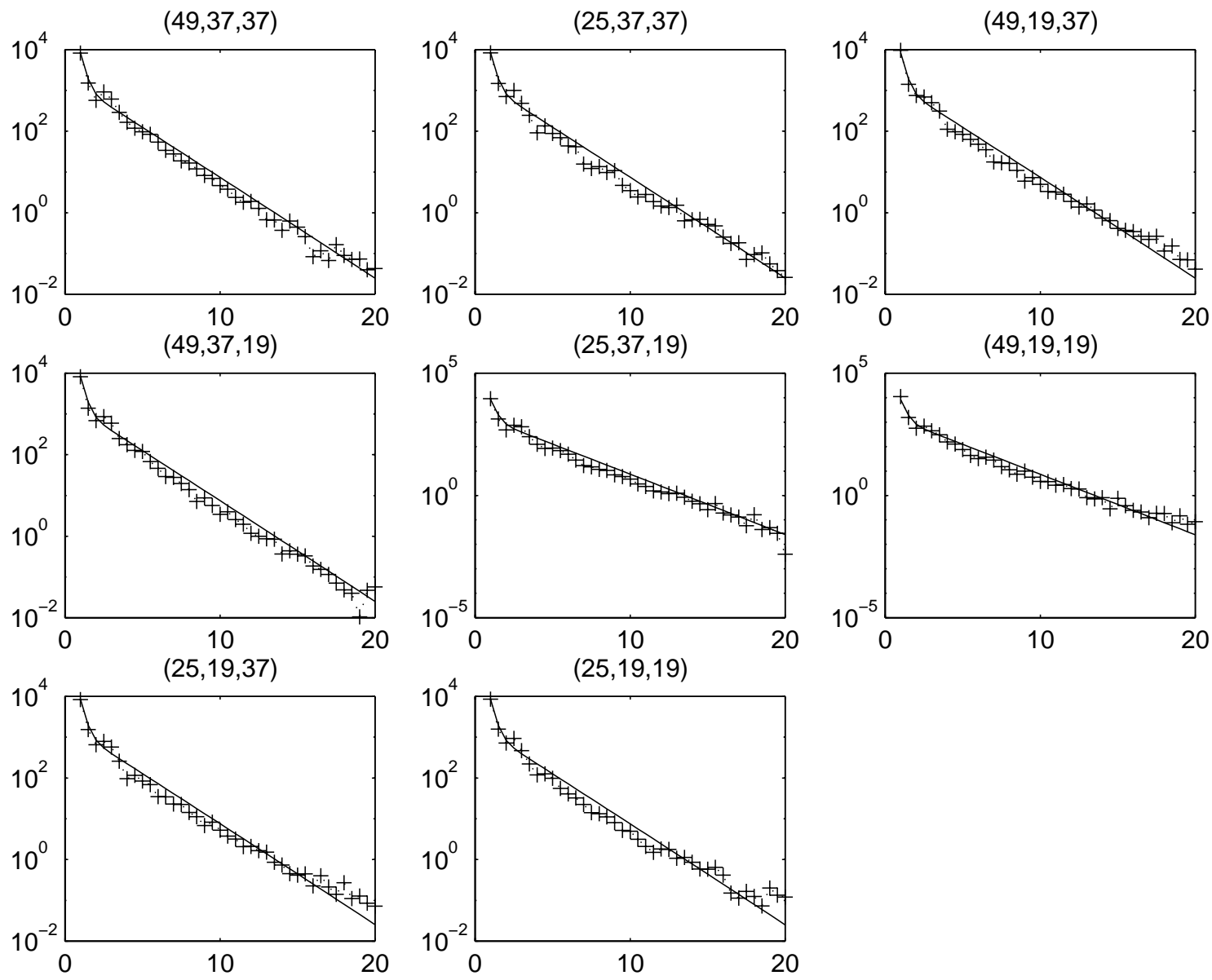

Figure 5. The comparison of the measured point spread function profiles ('+') and the exponential fitting function (solid line). The numbers above each plot are the $(\mathrm{x}, \mathrm{y}, \mathrm{z})$ coordinates of the point source. The $\mathrm{x}$-axis is the distance $(\mathrm{mm})$ from the point source, and the $\mathrm{y}$-axis is the mean activity.

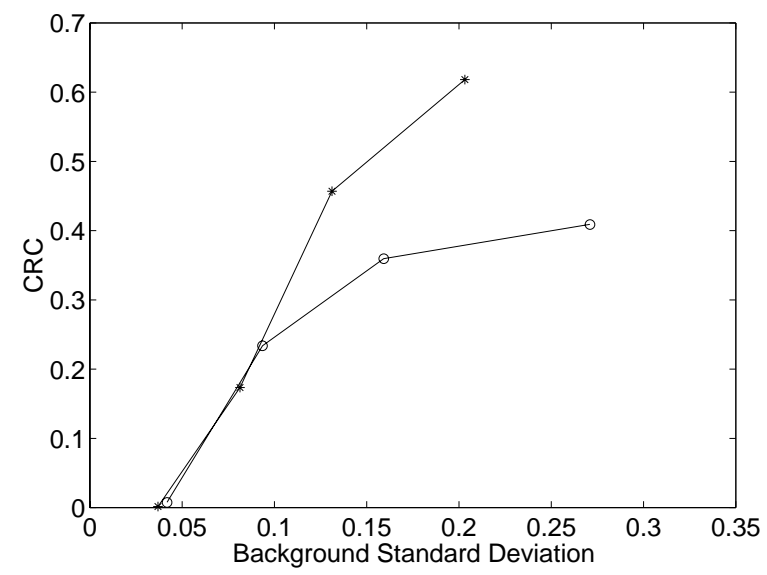

Figure 6. The comparison of CRC vs. background standard deviation plots for images reconstructed with ('*') and without ('o') modeling of crystal scatters. The points on the curves were obtained with different smoothing parameters. 
We have also theoretically analyzed the noise propagation from the estimate scatter sinogram into the final reconstructed image. The results show that the noise propagated from the estimated scatter sinogram is equivalent to increasing the noise variance in each LOR by a factor of $1+\sigma_{\widehat{s}_{i}}^{2} / \bar{y}_{i}$. If we assume the scatter fraction for each LOR is the same, then this factor is constant for all LORs, and it provides an easy way to calculate the required number of events in the Monte Carlo scatter simulation for a given noise level in reconstruction. This noise analysis is applicable to other scatter estimation methods, provided that an estimate of the covariance of the estimated scatter sinogram is available.

\section{Acknowledgments}

The authors would like to thank Robort L. Smith of the Center for Functional Imaging for proofreading the manuscript.

This work was supported by the U.S. Department of Health and Human Services, National Institutes of Health under grant P01 HL25840 and by the Director, Office of Science, Office of Biological and Environmental Research, Medical Sciences Division of the US Department of Energy under contract DE-AC03-76SF00098.

\section{Appendix}

\section{A Validation of (12) Using Monte Carlo Simulation}

We conducted a computer Monte Carlo simulation to validate (12). We simulated a one ring PET system with only 40 detectors so that (12) can be easily computed. The ring diameter was $172 \mathrm{~mm}$. The number of LORs per view was 32 . The image was $32 \times 323 \mathrm{~mm}$ square pixels. The phantom was a uniform disk as shown in Fig. A1. The projection data were generated by forward projecting the noise free image and adding a uniform scatter sinogram. The total number of true events was 100000 , and the total number of scatter events was 50000 . We reconstructed the noise free projection data with noisy scatter sinogram estimate. The Monte Carlo scatter estimate was simulated using a Poisson random number generator. The noise level was controlled by the total number of events in the estimated scatter sinogram. At each noise level, we generated 1000 independent noisy scatter estimates, and used it to reconstruct the noise free projection data. A variance image is then computed from these 1000 independently reconstructed images. Since the projection data were noise free, the variance in the reconstructed images was solely due to the noise in the scatter estimate.

Fig. A2 shows the comparison of the standard deviation images that were computed from (12) and that from 1000 reconstructions. The noise level was 50000 events. Since (12) does not model the non-negativity constraint used in the reconstruction, it cannot predict the variance in the zero background region. Fortunately, we are less interested in the background, so we masked both variance images with the true phantom image. The images show that within the support region of the disk phantom, the variance computed 

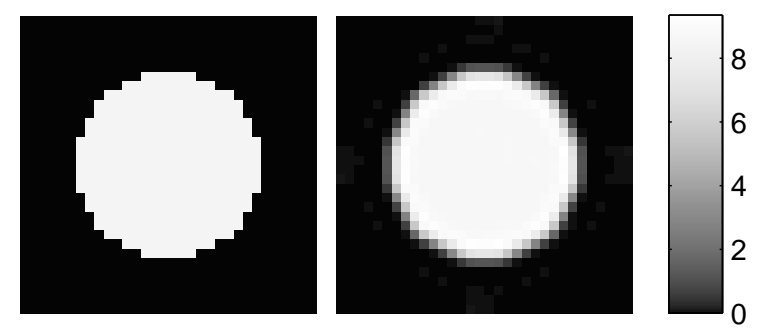

Figure 1. The disk phantom image (left) used in the computer simulation and the mean image of 1000 independent reconstructions with noisy scatter estimate (right).
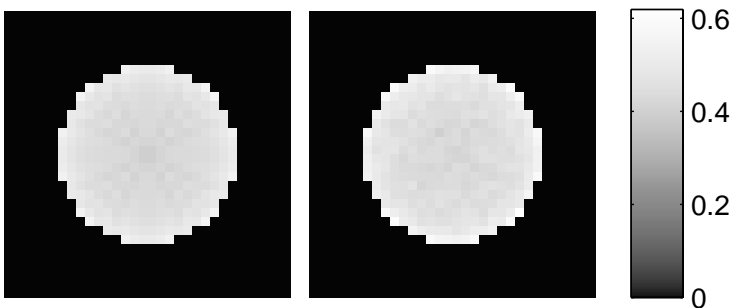

Figure 2. The standard deviation images that were obtained from theoretical approximation (12) (left) and that obtained from 1000 independent reconstructions (right). Both images are masked with the disk phantom image.

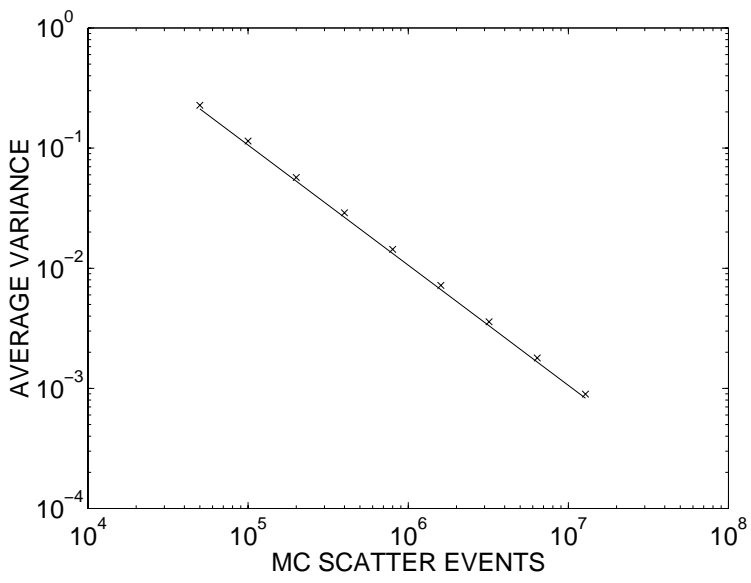

Figure 3. The average variance of all pixels within the disk as a function of the number of Monte Carlo simulated scatter events. Solid line denotes the theoretical predictions and ' $x$ ' denotes the results from 1000 reconstructions.

from (12) is quite close to that obtained from 1000 reconstructions.

In Fig. A3 we plot the average variance of all the pixels within the disk as a function of the noise level (the number of Monte Carlo simulated scatter events). It shows that the variance in reconstruction is inversely proportional to the count level of the Monte Carlo scatter simulation and that the theoretical approximation matches the Monte 
Carlo results reasonably well.

\section{References}

[1] P. Virador, W. Moses, and R. Huesman, "Reconstruction in PET cameras with irregular sampling and depth of interaction capability," IEEE Transactions on Nuclear Science, vol. 45, pp. 1225$1230,1998$.

[2] W. W. Moses, S. E. Derenzo, C. L. Melcher, and R. A. Manente, "A room temperature LSO/PIN photodiode PET detector module that measures depth of interaction," IEEE Transactions on Nuclear Science, vol. 42, pp. 1085-1089, 1995.

[3] R. H. Huesman, G. J. Klein, W. W. Moses, J. Qi, B. W. Reutter, and P. R. G. Virador, "List mode maximum likelihood reconstruction applied to positron emission mammography with irregular sampling," IEEE Transactions on Medical Imaging, vol. 19, pp. 532-537, 2000.

[4] D. L. Bailey and S. R. Meikle, "A convolution-subtraction scatter correction method for 3D PET," Physics in Medicine and Biology, vol. 39, pp. 411-424, 1994.

[5] A. Dempster, N. Laird, and D. Rubin, "Maximum likelihood from incomplete data via the EM algorithm," Journal of Royal Statistical Society, Series B, vol. 39, pp. 1-38, 1977.

[6] L. Shepp and Y. Vardi, "Maximum likelihood reconstruction for emission tomography," IEEE Transactions on Medical Imaging, vol. 1, pp. 113-122, 1982.

[7] K. Lange and R. Carson, "EM reconstruction algorithms for emission and transmission tomography," Journal of Computer Assisted Tomography, vol. 8, pp. 306-316, 1984.

[8] S. Grootoonk, T. J. Spinks, D. Sashin, N. M. Spyrou, and T. Jones, "Correction for scatter in 3D brain PET using a dual energy window method," Physics in Medicine and Biology, vol. 41, pp. 2757-2774, 1996.

[9] C. S. Levin, M. Dahlbom, and E. J. Hoffman, "A Monte Carlo correction for the effect of Compton scattering in 3-D PET brain imaging," IEEE Transactions on Nuclear Science, vol. 42, pp. 1181-1185, 1995.

[10] J. M. Ollinger, "Model-based scatter correction for fully 3D PET," Physics in Medicine and Biology, vol. 41, pp. 153-176, 1996.

[11] C. C. Watson, "New, faster, image-based scatter correction for 3D PET," IEEE Transactions on Nuclear Science, vol. 47, pp. 1587-1594, 2000.

[12] J. A. Fessler, "Mean and variance of implicitly defined biased estimators (such as penalized maximum likelihood): Applications to tomography," IEEE Transactions on Image Processing, vol. 5, pp. 493-506, 1996.

[13] E. Mumcuoglu, R. Leahy, S. Cherry, and E. Hoffman, "Accurate geometric and physical response modeling for statistical image reconstruction in high resolution PET," in Proceedings of IEEE Nuclear Science Symposium and Medical Imaging Conference, pp. 1569-1573, Anaheim, CA, 1996.

[14] J. Qi, R. M. Leahy, S. R. Cherry, A. Chatziioannou, and T. H. Farquhar, "High resolution 3D Bayesian image reconstruction using the microPET small animal scanner," Physics in Medicine and Biology, vol. 43, pp. 1001-1013, 1998.

[15] A. J. Reader, S. Ally, F. Bakatselos, R. Manavaki, R. J. Walledge, A. P. Jeavons, P. J. Julyan, S. Zhao, D. L. Hastings, and J. Zweit, "Regularised one-pass list-mode EM algorithm for high resolution 3D PET image reconstruction into large arrays," in Proc. IEEE Nuc. Sci. Symp. Med. Im. Conf., pp. M11-2, 2001.

[16] R. J. Ott, K. Erlandsson, M. A. Flower, and A. Reader, "Image-space 3D scatter correction following list mode acquisition with a large-area positron camera," in Proceedings of IEEE Nuclear Science Symposium and Medical Imaging Conference, Toronto, ON, Canada, 1998.

[17] A. J. Reader, S. Zhao, P. J. Julyan, D. L. Hastings, and J. Zweit, "Adaptive correction of scatter and random events for 3-D backprojected PET data," IEEE Transactions on Nuclear Science, vol. 48, pp. 1350-1356, 2001. 
[18] K. Lange, M. Bahn, and R. Little, "A theoretical study of some maximum likelihood algorithms for emission and transmission tomography," IEEE Transactions on Medical Imaging, vol. 6, pp. 106-114, 1987. 